\title{
Percepción social de acoso sexual en el trabajo
}

\author{
Yolanda NAVARRO ABAL \\ Departamento de Psicología Clínica, Experimental y Social \\ Universidad de Huelva \\ yolanda.navarro@dpsi.uhu.es \\ José Antonio CLIMENT RODRÍGUEZ \\ Departamento de Psicología Clínica, Experimental y Social \\ Universidad de Huelva \\ jose.climent@dpsi.uhu.es \\ María José RUIZ GARCÍA \\ Departamento de Anton Menger \\ Universidad de Huelva \\ mruiz1354@icahuelva.es
}

Recibido: 1-03-2011

Aceptado: 28-03-2011

\section{RESUMEN}

El acoso sexual en el trabajo, es un fenómeno social que está saliendo a la luz en los últimos años. Las estadísticas informan sobre la escasez de denuncias que se producen a pesar de ser un fenómeno que cada vez se hace más visible en nuestra sociedad. El objetivo del presente estudio es analizar la percepción e información que existe relacionado con el fenómeno del acoso sexual como variable relevante para la realización de programas de prevención tanto en futuros profesionales como en los profesionales que forman parte de un entorno laboral. Se administró un cuestionario a una muestra de 65 alumnos/as de la Titulación de Psicología y 15 alumnos/as de la Titulación de Ciencias del Trabajo de la Universidad de Huelva, y 44 personas que se encuentran inmersos en el mundo laboral. Los resultados indican falta de formación e información sobre el fenómeno del acoso sexual así como de sensibilización especialmente hacia la víctima.

Palabras claves: acoso sexual en el trabajo, percepción, programas de prevención, sensibilización

\section{Social perception of sexual harassment in the work}

\begin{abstract}
The sexual harassment in the work, is a social phenomenon that is going out to the light in the last years. The statistics inform about the shortage of denunciations that they take place in spite of being a phenomenon that every time becomes more visible in our company. The aim of the present study is to analyze the perception and information that exists related to the phenomenon of the sexual harassment as relevant variable for the accomplishment of programs of prevention both in professional futures and in the professionals who form a part of a job environment. One administered a questionnaire to a sample of 65 pupils / aces of the Qualifications of Psychology and 15 pupils / aces of the University of Huelva, and 44 persons who are immersed in the labour world. The results indicate lack of formation and information about the phenomenon of the sexual harassment as well as of awareness specially towards the victim.
\end{abstract}


Keywords: Sexual harassment in the work, perception, programs of prevention, awareness

REFERENCIA NORMALIZADA

Navarro Abal, Y., Climent Rodríguez, J. A. y Ruíz Rodríguez, Mª J. (2012). Percepción social de acoso sexual en el trabajo. Cuadernos de Relaciones Laborales Vol. 30, núm. 2, p. 541-561.

SUMARIO: 1. Descripción y conceptualización de acoso sexual en el trabajo. 2. Objetivo general. 3. Método. 3.1. Muestra. 3.2. Instrumento. 3.3. Procedimiento. 4. Resultados. 5. Discusión. 6. Referencias bibliográfiacs.

\section{Descripción y conceptualización de acoso sexual en el trabajo}

Aunque el término, y la preocupación social por sus consecuencias, son relativamente recientes, el acoso sexual es un desgraciado, viejo y persistente problema en cualquier escenario en el que se desarrollan las relaciones humanas, y, desde luego en el contexto laboral. Claramente se trata de una forma de violencia, que puede tener connotaciones tanto de violencia física como psicológica. Necesariamente resulta una forma de discriminación, que ocurre cuando una conducta no deseada de naturaleza sexual interfiere con el trabajo individual. Estadísticamente es un comportamiento que, aunque deja víctimas de ambos sexos, afecta mayoritariamente a mujeres en su entorno de trabajo.

Múltiples son los autores e instituciones sociolaborales que se han ocupado de estudiar y denunciar el acoso sexual en el trabajo en las dos últimas décadas, conceptualizándolo de manera muy análoga. Así, Stockdale (1996), señala que existe cierto consenso para definir el acoso sexual en el trabajo como "una atención sexual indeseada"; concluyendo que, al menos, una de cada diez y posiblemente incluso una de cada dos mujeres experimenta acoso sexual en el trabajo a lo largo de su vida laboral. La propia Organización Internacional del Trabajo (OIT), ya en 1997 indicaba que inclusive cuando en una sociedad haya algunos que nieguen la existencia del problema, éste se ve confirmado por quienes son víctimas, lo cual quiere decir que ignorar que exista no significa que no ocurra. Más aún, señala que se disponía ya entonces de un creciente corpus de investigaciones empíricas, incluida jurisprudencia, que documentaba la incidencia y existencia del acoso sexual en el trabajo. De acuerdo con la Comisión de Igualdad de Oportunidades de Empleo de Estados Unidos (1980) el acoso sexual es una forma de discriminación sexual que ocurre cuando una conducta física o verbal no deseada de naturaleza sexual afecta el trabajo individual, interfiere de manera irracional con el desempeño individual en el trabajo o crea un ambiente de trabajo hostil u ofensivo. Para Castillo (1997), el acoso sexual constituye una de las formas más comunes de violencia contra la mujer, y se da especialmente en los centros de trabajo, de estudio, y de atención profesional (médica, psicológica, odontológica, otros); aunque, reconoce que puede afectar tanto a hombres como a mujeres, pero señala que de hecho es más extendida contra las mujeres, como expresión de los mecanismos de poder que sobre ellas ejercen tradicionalmente los hombres desde la instauración del sistema patriarcal, que crea la inequidad del género. 
Desde la Secretaria de la Mujer de la Unión General de Trabajadores (1994), se afirma que el acoso sexual es, por encima de todo, una manifestación de relaciones de poder, estando las mujeres mucho más expuestas a ser víctimas del acoso sexual precisamente porque carecen de poder en el ámbito laboral, aunque también corren peligro de padecer semejante conducta cuando se las percibe como competidoras por el poder. Precisamente, desde esta institución sindical se determina que el acoso sexual es la conducta verbal o física de carácter sexual u otros comportamientos fundamentados en el sexo, que vulneran la dignidad del hombre y la mujer en el trabajo, y que son consideradas ofensivas o no deseadas por quien las padece, determinando que, a principios del siglo XXI un $18^{\prime} 3 \%$ de mujeres trabajadoras españolas han sufrido alguna vez acoso sexual. Este mismo estudio denunciaba que el $62 \%$ de las mujeres que se ven en situación de acoso intentan evitar al acosador, incluso en ocasiones optan por abandonar el trabajo como única forma de acabar con el acoso; en otros casos la baja laboral ocurre por depresión a causa del estrés producido por la situación de acoso; solo el 3\% de las acosadas pusieron una denuncia ante las autoridades judiciales. Por último, el 50.4\% ha estado en situación de acoso ambiental a través de chistes y comentarios de contenido sexual, por parte de sus compañeros o superiores.

Otros datos de interés sobre la incidencia del acoso sexual en el trabajo en nuestro país lo proporcionan el informe del año 2000 de Comisiones Obreras, según el cual el $18 \%$ de las trabajadoras españolas se enfrentan a situaciones en las que los jefes y colegas invaden su espacio físico, con insinuaciones sexuales no deseadas, o son el objeto de chantajes de jefes, que condicionan su futuro en el trabajo a la aceptación de una relación sexual. Además, el 30\% de los incidentes de acoso han tenido como protagonistas una trabajadora sin contrato. Otro dato significativo, que corrobora los datos de otras investigaciones e informes, es que el $40 \%$ de las víctimas están separadas o divorciadas, siendo el estado civil y tener o no pareja estable un factor que correlaciona con la mayor o menor probabilidad de sufrir un episodio de acoso sexual, más que otros factores relacionados con el atractivo físico, edad, experiencia laboral y otros (Pernas, Olza y Román, 2000).

Lo cierto es que una revisión de los elementos definitorios del término acoso sexual en el trabajo coinciden en señalar que se trata de una conducta de naturaleza sexual, que afecta la dignidad de mujeres y hombres, y que resulta ingrata, irrazonable y ofensiva para quien la recibe. Otros aspectos coincidentes en la mayor parte de las definiciones determina que el rechazo de la persona acosada a esa conducta, $o$ su sumisión a ella, se emplea explícita o implícitamente como base para una decisión que afecta al trabajo de esa persona. El tercer elemento común a la mayoría de las conceptualizaciones de acoso sexual, señala que se trata de una conducta que crea un ambiente de trabajo intimidatorio, hostil o humillante para quien la recibe. En relación a esto último señalar que autores como Piñuel y Zabala (2003) señalan que el acoso sexual no siempre persigue un beneficio carnal, sino que puede simplemente crear un ambiente de temor, coacción y denigración humillante para la víctima. 
El acoso sexual, según Barak, Fisher y Huston (1992), puede ser clasificado como acoso de género, solicitud de actividad sexual explícita con promesa de premio o castigo, y asalto o imposición sexual. Para Livingston (1982), el fenómeno del acoso sexual no se puede explicar a través de un modelo simple, señalando que el poder económico y social de acosadores y víctimas influye en la ocurrencia y severidad del acoso, así como en el tipo de respuestas y la efectividad de las acciones de la víctima. Por su parte, Tangri, Burt y Johson (1982) citan 3 modelos teóricos explicativos del acoso sexual: El modelo biológico establece que el acoso sexual no es más que la manifestación de la atracción sexual, que depende de la carga biológica de las personas. Por su parte, el modelo organizacional pone énfasis en los factores estructurales de cualquier organización, organigrama empresarial, condiciones de trabajo, proporción de personas de uno y otro género, funciones desempeñadas por los miembros de la organización. En esta línea, Fitzgerald et. al. (1997), plantea que la cultura grupal y el clima organizativo son claves para comprender el marco en el que se desarrolla el acoso sexual. Por su parte un modelo socio-cultural de explicación del acoso, lo considera como un "producto" derivado de las normas actuales, valores, estereotipos y creencias que enfatizan el papel dominador del hombre sobre la mujer.

Según Ardouin, Bustos, Jarpa y Oliveros (2001), tres factores son fundamentales para abordar este problema: diferencias culturales, que señalan que la agresión sexual de los hombres puede ser algunas veces producto de una mala interpretación de los contactos femeninos, en cuanto a considerar conductas amistosas de las mujeres como expresiones de interés sexual. Al respecto refieren un estudio de Menon, S. y Kanebar, S (1992), realizado en la India, quienes encontraron que allí se puede considerar que el acoso sexual a mujeres es sintomático, ya que se espera que sea el hombre el que tome la iniciativa, mientras la mujer debe adoptar una postura sumisa. Factores del ambiente de trabajo, que se refieren, por ejemplo, a que a menor status de la víctima en la organización en comparación con la del acosador, mayor será la intensidad del acoso, o a que a mayor status del acosador es más probable que la mujer perciba que por su status se acerca para explotarla sexualmente. Un tercer factor lo constituye la respuesta de la Víctima. Los estudios en este sentido señalan que las víctimas que no actúan contra el acosador tienen más probabilidades de ser censuradas y culpadas que aquellas que protestan.

Se pueden distinguir dos tipos básicos de acoso sexual, en función de que exista o no un elemento de chantaje. Acoso quid pro quo: lo que se produce es un chantaje sexual. Se fuerza a una persona a elegir entre someterse a los requerimientos sexuales o perder o ser perjudicados ciertos beneficios o condiciones de trabajo. $\mathrm{O}$ acoso que crea un ambiente de trabajo hostil: cuando existe una conducta que crea un ambiente de trabajo humillante, hostil o amenazador para el acosado.

Otros autores prefieren clasificar el acoso en base a conductas verbales, conductas físicas y otras conductas, añadiendo a cada una de ellas, según su gravedad, el adjetivo de leve, moderado o grave. Así, chistes, piropos ofensivos, reiteración en la petición de citas serían conductas verbales de tipo leve, tocar, acariciar, besar, serían catalogadas como conductas físicas graves, y así sucesivamente. En este 
sentido, la Secretaría de la Mujer de la Unión del Personal Civil de la Nación (UPCN) (1997) en Buenos Aires, en un estudio llevado a cabo con trabajadoras del sector público, determinaron cinco niveles de conductas de acoso sexual, para las que se tuvo en cuenta el tipo de interacción (verbal - no verbal), el contenido del mensaje (menos o más coercitivo) y la implicación o no de contacto físico:

Nivel 1) Acoso leve, verbal: chistes, piropos, conversaciones de contenido sexual.

Nivel 2) Acoso moderado, no verbal y sin contacto físico: Miradas, gestos lascivos, muecas.

Nivel 3) Acoso medio, fuerte verbal: Llamadas telefónicas y/o cartas, presiones para salir o invitaciones con intenciones sexuales.

Nivel 4) Acoso fuerte, con contacto físico: Manoseos, sujetar o acorralar.

Nivel 5) Acoso muy fuerte: Presiones tanto físicas como psíquicas para tener contactos íntimos.

Otras clasificaciones, ponen el acento en la intensidad o gravedad de la conducta de acoso, diferenciando cuatro tipologías:

A) Requerimiento de favores sexuales, acompañado de promesas explícitas o implícitas de un trato preferencial si se accede al requerimiento o bien de amenazasen caso de no acceder.

$B$ ) Contacto físico de carácter sexual, generalmente acompañado de comentarios o gestos ofensivos no deseados por la acosada, sin empleo de fuerza o intimidación.

C) Invitaciones impúdicas o comprometedoras o uso de material pornográfico en el centro de trabajo.

D) Bromas sobre el sexo, o abuso del lenguaje con comentarios sobre la apariencia, o con algunas observaciones sugerentes y desagradables.

Autores como Ardouin, Bustos, Jarpa y Oliveros (2001) plantean que las diferencias de género en la percepción de acoso sexual son producto del mayor número de experiencias negativas que tienen las mujeres con relación al trabajo y con relación al sexo; aún cuando hombres y mujeres reconocen el acoso, difieren en el momento de juzgar la conducta como acoso. Así, la mujer percibe más cantidad de conductas como acoso (hasta las más sutiles) que el hombre. Además existen diferencias entre los motivos percibidos por ambos sexos para el acoso sexual, considerando los hombres sobre todo el atractivo sexual y las mujeres, otros factores además del anterior, del tipo poder económico o estatus.

Por último, en cuanto a las consecuencias para la víctima y la organización que lo padece, la OIT (1997) señala que estrés emocional, humillación, depresión, ira, impotencia, fatiga, enfermedad física, son algunos de los potencialmente graves efectos a nivel individual. Niveles elevados de tensión en el trabajo, insuficiente colaboración en el trabajo en equipo, bajo rendimiento, absentismo, disminución de 
la productividad, son algunos efectos sobre la organización en la que se producen situaciones de acoso sexual.

Así mismo, es necesario resaltar que una de las mayores consecuencias de las mujeres que se han negado a las conductas acosadoras del agresor son posteriormente objetos de acoso moral sufriendo todos los comportamientos así como sus consecuencias biopsicosociales.

Los pocos estudios que existen relacionados con el acoso sexual se han realizado en personas que se encuentran inmersas en el mundo laboral y en las que se ha valorado si han existido conductas que se hayan podido percibir como indicadoras de acoso. En este trabajo se intenta analizar aquellas deficiencias formativas y actitudinales que nos encontramos en el alumnado con la idea básica de poder desarrollar acciones formativas en las cuales se adquieran conocimientos y competencias suficientes tanto para la prevención como para el establecimiento de estrategias que permitan paliar este tipo de comportamientos.

\section{Objetivo general}

El objetivo general de esta investigación es dar a conocer y sensibilizar a la sociedad sobre las conductas relacionadas con el acoso sexual en el trabajo. Para ello se analizará la percepción que existe de las mismas con el fin de que puedan ser de utilidad a la hora de elaborar programas para la prevención e intervención de estas conductas a nivel individual, grupal y en el seno de las organizaciones laborales.

\section{Método}

\subsection{Muestra}

En la realización del presente estudio se ha utilizado una muestra compuesta por un total de 126 personas distribuidas de la siguiente forma; 67 alumnos que cursan $5^{\mathrm{o}}$ de la Titulación de Psicología de la Universidad de Huelva, 15 alumnos que cursan $5^{\circ}$ de la licenciatura de C.C. del Trabajo de la Universidad de Huelva y 44 personas con estudios universitarios que se encuentran insertados en el mundo laboral. El $77 \%(n=97)$ son mujeres y el $23 \%(n=29)$ hombres. La media de edad de la muestra es de 29 años, perteneciendo el $70 \%(n=88)$ al rango de edad entre 23 y 29 años y el $30 \%(n=38)$ entre 29 y 41 años.

\subsection{Instrumentos}

Para llevar a cabo este análisis, se elaboró un cuestionario que incluía tres bloques diferenciados:

Bloque I. Variables sociodemográficas.

Bloque II. Compuesto por 10 preguntas de respuesta abierta relacionadas con el concepto de acoso, perfil de la víctima y del agresor, prevención del acoso y actitud 
ante el acoso. En cada una de las preguntas existía un apartado denominado "comentarios" en donde la persona podía explicar o aclarar su respuesta.

Bloque III. Compuesto por 14 preguntas cerradas, en una escala de respuesta tipo likert, de cinco puntos, que oscilan desde la opción nunca (0) a la opción siempre (5). El contenido de estas preguntas hace referencia a conductas verbales y no verbales indicadoras del acoso sexual en el trabajo. Así mismo, para que cada persona pudiera aclarar alguna duda con respecto a sus respuestas cada uno de los ítems contaba con un apartado denominado "comentario" en donde la persona podría aclarar la respuesta dada.

\subsection{Procedimiento}

La administración de las pruebas se realizó siguiendo dos procedimientos diferentes en función del grupo muestral: por un lado, el alumnado de la $5^{\circ}$ de la Titulación de Psicología y de $5^{\circ}$ de la Titulación de C.C. del Trabajo de la Universidad de Huelva rellenó los instrumentos en el propio aula en donde se recibía la docencia; por otro lado, a la muestra constituida por los trabajadores en activo, dada la diversidad de procedencia, se le aplicaron las pruebas de una manera individualizada. Para evitar los sesgos referentes a la deseabilidad social se les explicaba a los participantes que iban a participar en una investigación pero se obviaba explicar el objetivo de la misma.

\section{Resultados}

A continuación exponemos los resultados obtenidos que han sido estructurados en dos apartados debido a las características de la prueba de evaluación. Por un lado, se han obtenido los resultados relacionadas con las preguntas abiertas (Bloque II) en las que se valoraba el concepto de acoso, perfil de la víctima y del agresor, prevención del acoso y actitud ante el acoso. Para ello se realizó un análisis de los ítems específicos de respuestas abiertas que conformaban el cuestionario. Con el objetivo de obtener respuestas lo más concretas posibles que aportasen una mayor información a nuestra investigación, elaboramos diferentes categorizaciones de dichas cuestiones. Para ello se elaboró una categorización en función a los datos obtenidos.

En segundo lugar, se exponen los resultados del análisis de los ítems correspondiente a las preguntas cerradas con formato de respuesta de escala tipo likert (Bloque III).

\section{Bloque II}

Con respecto al análisis de las variables cualitativas, y una vez llevada a cabo la categorización, los valores que adoptan las variables son: 
Ítem 1. ¿Qué consideras que es el acoso sexual?

- Conducta con connotaciones sexuales $(33,33 \%)(n=42)$

- Conducta que atenta contra la libertad de la persona $(16,66 \%)(n=21)$

- Conducta sexual que incomoda e intimida a la persona $(26,98 \%)(n=34)$

- Actitud intimidatoria $(15,87 \%)(\mathrm{n}=20)$

- $\operatorname{Otras}(7,14 \%)(\mathrm{n}=9)$

Con respecto al concepto de acoso sexual, los resultados muestran su asociación clara a conductas sexuales siendo un total del $60,31 \%(n=76)$ de los participantes los que así lo indican así como a características intimidatorias como queda reflejada en el 42,85\% (n=54). Así mismo, el 16,66\% 8n=21) lo relacionan con conductas no específicamente sexuales pero en los que destacan su influencia en el derecho a la libertad de la persona.

Ítem 2. ¿Cuándo consideras que es más habitual el acoso?

- Cuando la víctima es atractiva $(38,09 \%)(n=48)$

- Cuando no hay igualdad de poder $(21,42 \%)(n=27)$

- Cuando hay menos recursos económicos $(18,25 \%)(n=23)$

- $\operatorname{Otras}(22,22 \%)(n=28)$

Con respecto a las variables consideradas por los participantes como más determinantes en el desarrollo del acoso hay que destacar como el porcentaje mayor, el $38,09 \%(\mathrm{n}=48)$ se asocia al atractivo físico de la persona, seguido por orden de importancia la falta de igualdad de poder, el $21,42 \%(n=27)$ y la falta de recursos económicos, el 18,25\% $(\mathrm{n}=23)$.

Ítem 3. ¿Crees que se puede producir el acoso sexual entre dos iguales?

- $\mathrm{Si}$, aunque no es lo habitual $(26,19 \%)(\mathrm{n}=33)$

- No, suele ser entre personas con más poder $(42,85 \%)(n=54)$

- $\mathrm{Si}$, siempre que alguien tenga una situación más favorecedora $(18,25 \%)$ $(\mathrm{n}=23)$

- $\operatorname{Otras}(12,69 \%)(\mathrm{n}=16)$

En relación a la manifestación de acoso sexual entre iguales, los datos indican que un $42,58 \%(n=54)$ consideran que no es posible, un $26,19 \%(n=33)$ consideran que es posible y un $18,25 \%(\mathrm{n}=23)$ considera que podría ser siempre que se diera la condición de desigualdad de condiciones. 
Ítem 4. ¿Crees que el acoso sexual se podría evitar?

- Si, si la víctima se opusiera desde el principio $(30,95 \%)(n=39)$

- $\quad \mathrm{Si}$, si la ley fuera más dura $(13,49 \%)(\mathrm{n}=17)$

- $\quad \mathrm{Si}$, si fuera aceptado socialmente $(15,07 \%)(\mathrm{n}=19)$

- No, porque son personas psicópatas que saben lo que hacen $(15,87 \%)$ $(\mathrm{n}=20)$

- No, porque siempre se realiza en personas más pobres y con menos recursos que no se pueden defender $(17,46 \%)(\mathrm{n}=22)$

- $\operatorname{Otras}(7,14 \%)(n=9)$

En cuanto a la evitación del acoso sexual el 30,95\% $(n=39)$ considera que sería posible si la víctima se opusiera desde el principio, el $13,49 \%(\mathrm{n}=17)$ si la ley fuera más dura, el 15,07\% $(\mathrm{n}=19)$ opina que está determinado por la aceptación social, el $15,87 \%(\mathrm{n}=20)$ considera que no es posible ya que son psicópatas, y el $17,46 \%$ $(\mathrm{n}=22)$ no es posible debido a las condiciones desfavorecedoras de la víctima.

Ítem 5. ¿Cuál es el perfil del agresor/a?

- Un hombre psicópata $(22,22 \%)(\mathrm{n}=28)$

- Un hombre vicioso $(26,98 \%)(\mathrm{n}=34)$

- Un hombre adicto al sexo $(14,28 \%)(\mathrm{n}=18)$

- Una persona mala $(21,42 \%)(n=27)$

- Una persona más vulnerable a la que provocan que eso ocurra $(5,55 \%)$ $(\mathrm{n}=7)$

- $\operatorname{Otras}(9,52 \%)(\mathrm{n}=12)$

Analizando los resultados obtenidos en cuanto al perfil del agresor destacamos como un total de $63,48 \%(\mathrm{n}=80)$ considera que la persona acosadora es un hombre aunque con algunos aspectos diferenciadores: el $26,99 \%(n=34)$ considera que es un vicioso, el $22,22 \%(\mathrm{n}=28)$ un psicópata y el $14,28 \%(\mathrm{n}=18)$ un adicto al sexo. Así mismo, un $21,42 \%(n=27)$ considera que el perfil se caracteriza por ser una persona mala sin identificación del sexo y un $5,55 \%(\mathrm{n}=7)$ opina que son personas que realizan estas conductas porque hay otras personas que los provocan.

Ítem 6. ¿Cuál consideras que es el perfil de la víctima?

- Una mujer "ligera" $(21,42 \%)(n=27)$

- Una mujer atractiva $(34,12 \%)(n=43)$

- Una mujer que tiene pocos recursos económicos $(18,25 \%)(n=23)$

- Persona (hombre o mujer) con menos recursos $(19,04 \%)(n=24)$

- $\operatorname{Otras}(7,14 \%)(\mathrm{n}=9)$

En relación al perfil de la víctima, un $73,79 \%(\mathrm{n}=93)$ considera que la víctima es mujer aunque se diferencias algunas características: el $34,12 \%(\mathrm{n}=43)$ lo asocia al atractivo de la víctima, el $21,42 \%(n=27)$ considera que se refiere a mujeres "ligeras" y el 18,25\% (n=23) lo asocia a pocos recursos económicos. Así mismo, el 
$19,04 \%(\mathrm{n}=24)$ considera que la víctima puede ser hombre o mujer pero que la característica esencial es la diferencia de recursos con respecto al agresor.

Ítem 7. ¿Has conocido a alguien que haya sufrido acoso?

$\begin{array}{ll}\text { - } & \text { Si }(18,25 \%)(\mathrm{n}=23) \\ \text { - } & \text { No }(81,74 \%)(n=103)\end{array}$

El $81,74 \%(\mathrm{n}=103)$ de los participantes no ha conocida ninguna persona que haya sufrido acoso, siendo el $18,25 \%(\mathrm{n}=23)$ los que afirman conocer a alguna persona.

Ítem 8. ¿Quién era el agresor?

- Jefe $(52,17 \%)(\mathrm{n}=12)$

- Compañero $(4,23 \%)(n=1)$

- Profesor $(30,43 \%)(n=7)$

- $\operatorname{Otras}(13,04 \%)(n=3)$

De $\operatorname{los} 18,25 \%(\mathrm{n}=23)$ de las personas que han conocido algún caso de acoso, el $52,17 \%(\mathrm{n}=12)$ afirma que el agresor era el jefe, el 30,43\% $(\mathrm{n}=7)$ un profesor, un $4,23 \%(n=1)$ la agresión procedía de algún compañero y un $13.04 \%(n=3)$ eran otras personas.

Ítem 9. ¿Se ha puesto en conocimiento el hecho? ¿A quién se puso en conocimiento?

- No $(73,91 \%)(\mathrm{n}=17)$

- Si $(13,04 \%)(n=3)$, amigos $(n=3)$

- $\operatorname{Otras}(13,04 \%)(n=3)$

De los 18,25\% $(\mathrm{n}=23)$ de las personas que conocen algún caso de acoso, el $73,91 \%(\mathrm{n}=17)$ manifiesta no haberlo puesto en conocimiento de nadie, un $13,04 \%$ $(n=3)$ afirma haberlo comunicado al entorno de los amigos y un $13,04 \%(n=3)$ considera haberlo confrontado con otras estrategias.

Ítem 10. Si fueses observador externo/a ¿qué crees que harias?

- Denunciar $(23,01 \%)(\mathrm{n}=29)$

- Hablar con el agresor $(28,57 \%)(\mathrm{n}=36)$

- Hablar con la víctima $(38,88 \%)(\mathrm{n}=49)$

- $\operatorname{Otras}(9,52 \%)(\mathrm{n}=12)$

Con respecto al comportamiento y actitud que tomarían en el caso de ser observadores, el 38,88\% $(\mathrm{n}=49)$ considera que hablaría con la víctima, el $28,57 \%(\mathrm{n}=36)$ hablaría con el agresor y el 23,01\% (n=29) denunciaría. 


\section{Bloque III}

Los resultados obtenidos con respecto al análisis de las variables cuantitativas son los siguientes:

1. Variable: Percepción de acoso sexual en el trabajo según los diferentes niveles de gravedad

1.1.Percepción de acoso sexual en el trabajo (Nivel 1, Acoso leve, verbal)

\begin{tabular}{|l|c|c|c|c|c|}
\hline \multicolumn{7}{|c|}{ Tabla 1. Nivel I: Acoso leve, verbal } \\
\hline & $\mathbf{0}$ & $\mathbf{1}$ & $\mathbf{2}$ & $\mathbf{3}$ & $\mathbf{4}$ \\
\hline Chistes & $\begin{array}{c}38,88 \% \\
(\mathrm{n}=49)\end{array}$ & $\begin{array}{c}29,36 \% \\
(\mathrm{n}=37)\end{array}$ & $\begin{array}{c}15 \% \\
(\mathrm{n}=19)\end{array}$ & $\begin{array}{c}11,90 \% \\
(\mathrm{n}=15)\end{array}$ & $\begin{array}{c}4,76 \% \\
(\mathrm{n}=6)\end{array}$ \\
\hline Piropos & $33,33 \%$ & $34,9 \%$ & $14,28 \%$ & $11,90 \%$ & $5,55 \%$ \\
& $(\mathrm{n}=42)$ & $(\mathrm{n}=44)$ & $(\mathrm{n}=18)$ & $(\mathrm{n}=15)$ & $(\mathrm{n}=7)$ \\
\hline Conversaciones de & $19.84 \%$ & $24,60 \%$ & $29 \%$ & $14,28 \%$ & $11.90 \%$ \\
contenido sexual & $(\mathrm{n}=25)$ & $(\mathrm{n}=31)$ & $(\mathrm{n}=37)$ & $(\mathrm{n}=18)$ & $(\mathrm{n}=15)$ \\
\hline
\end{tabular}

0(nunca); 1(casi nunca); 2 (a veces); 3 (muchas veces); 4 (siempre)

Como se observa en la Tabla 1, y en relación a los chistes como indicador de acoso sexual: el $38,88 \%(n=49)$ de los participantes consideran que un chiste nunca es un indicador, el $29,36 \%(n=37)$ que casi nunca, el $15 \%(n=19)$ que a veces, el $11,90 \%(\mathrm{n}=15)$ que muchas veces y el $4,76 \%(\mathrm{n}=6)$ que siempre. En cuanto a los piropos como indicador de acoso sexual, los resultados que se observan son: el $33,33 \%(n=42)$ considera que nunca, el $34,9 \%(n=44)$ que casi nunca, el $14,28 \%$ $(\mathrm{n}=18)$ que a veces, el 11,90\% $(\mathrm{n}=15)$ que muchas veces y el $5,55 \%(\mathrm{n}=7)$ que siempre. Finalmente, y con respecto a las conversaciones de contenido sexual, los resultados obtenidos son: el 19,84\% $(\mathrm{n}=25)$ considera que nunca se puede considerar acoso, el 24,60\% $(n=31)$ que casi nunca, el $29 \%(n=37)$ que a veces, el $14,28 \%$ $(\mathrm{n}=18)$ que muchas veces y el $11.90 \%(\mathrm{n}=15)$ que siempre.

1.2. Percepción de acoso sexual (Nivel 2, Acoso moderado, no verbal y sin contacto físico)

\begin{tabular}{|l|c|c|c|c|c|}
\hline \multicolumn{6}{|c|}{ Tabla 2. Nivel II: Acoso moderado, no verbal y sin contacto físico } \\
\hline & $\mathbf{0}$ & $\mathbf{1}$ & $\mathbf{2}$ & $\mathbf{3}$ & $\mathbf{4}$ \\
\hline Miradas & $5,55 \%$ & $17,46 \%$ & $28,57 \%$ & $26,19 \%$ & $22,22 \%$ \\
& $(\mathrm{n}=7)$ & $(\mathrm{n}=22)$ & $(\mathrm{n}=36)$ & $(\mathrm{n}=33)$ & $(\mathrm{n}=28)$ \\
\hline Gestos insinuantes & $7,14 \%$ & $15,87 \%$ & $33,33 \%$ & $23,01 \%$ & $20,63 \%$ \\
& $(\mathrm{n}=9)$ & $(\mathrm{n}=20)$ & $(\mathrm{n}=42)$ & $(\mathrm{n}=29)$ & $(\mathrm{n}=26)$ \\
\hline
\end{tabular}

0(nunca); 1(casi nunca); 2(a veces); 3(muchas veces); 4(siempre) 
En la Tabla 2 se describen los datos obtenidos en función del Nivel de acoso moderado obteniéndose los siguientes resultados: con respecto a la variable miradas, el 5,55\% ( $\mathrm{n}=7)$ considera que nunca es un indicador de acoso, el $17,46 \%$ $(n=22)$ que casi nunca, el 28,57\% $(n=36)$ cree que a veces, el $26,19 \%(n=33)$ opina que muchas veces mientras que el $22,22 \%(\mathrm{n}=28)$ cree que siempre. En cuanto a los gestos insinuantes, los resultados obtenidos son: el 7,14\% $(n=9)$ considera que nunca un gesto insinuante puede dar muestras de acoso, un $15,87 \%(\mathrm{n}=20)$ opina que casi nunca, un 33,33\% (n=42) piensa que a veces, el 23,01\% $(\mathrm{n}=29)$ manifiesta que muchas veces y un $20,63 \%(n=26)$ que siempre.

\subsection{Percepción del acoso sexual (Nivel 3, Acoso medio, fuerte verbal)}

\begin{tabular}{|l|c|c|c|c|c|}
\hline \multicolumn{7}{|c|}{ Tabla 3. Nivel III: Acoso medio, fuerte verbal } \\
\hline & $\mathbf{0}$ & $\mathbf{1}$ & $\mathbf{2}$ & $\mathbf{3}$ & $\mathbf{4}$ \\
\hline Llamadas telefónicas & $9,52 \%$ & $11,11 \%$ & $38,09 \%$ & $22,22 \%$ & $19,04 \%$ \\
& $(\mathrm{n}=12)$ & $(\mathrm{n}=14)$ & $(\mathrm{n}=48)$ & $(\mathrm{n}=28)$ & $(\mathrm{n}=24)$ \\
\hline Cartas (correos elec- & $11,90 \%$ & $17,46 \%$ & $28,57 \%$ & $23,01 \%$ & $19,04 \%$ \\
trónicos) & $(\mathrm{n}=15)$ & $(\mathrm{n}=22)$ & $(\mathrm{n}=36)$ & $(\mathrm{n}=29)$ & $(\mathrm{n}=24)$ \\
\hline Presiones para salir & $4,76 \%$ & $10,31 \%$ & $30,15 \%$ & $26,98 \%$ & $27,77 \%$ \\
& $(\mathrm{n}=6)$ & $(\mathrm{n}=13)$ & $(\mathrm{n}=38)$ & $(\mathrm{n}=34)$ & $(\mathrm{n}=35)$ \\
\hline Invitaciones con & $3,17 \%$ & $6,34 \%$ & $30,95 \%$ & $28,57 \%$ & $30,95 \%$ \\
intenciones sexuales & $(\mathrm{n}=4)$ & $(\mathrm{n}=8)$ & $(\mathrm{n}=39)$ & $(\mathrm{n}=36)$ & $(\mathrm{n}=39)$ \\
\hline
\end{tabular}

0(nunca); 1(casi nunca); 2 (a veces); 3 (muchas veces); 4 (siempre)

En relación a esta variable, la Tabla 3 muestra los resultados obtenidos: en cuanto a las llamadas telefónicas como indicadora de acoso sexual, el 9,52\% $(\mathrm{n}=12)$ afirma que nunca, el 11,11\% ( $\mathrm{n}=14)$ considera que casi nunca, el 38,09\% $(\mathrm{n}=48)$ piensa que a veces, el $22,22 \%(\mathrm{n}=28)$ opina que muchas veces y el $19,04 \%(\mathrm{n}=24)$ que siempre; sobre las cartas o correos electrónicos como conductas instigadoras de acoso los datos obtenidos son del 11,90\% $(\mathrm{n}=15)$ nunca, $17,46 \%(\mathrm{n}=22)$ casi nunca, $28,57 \%(\mathrm{n}=36)$ a veces, $23,01 \%(\mathrm{n}=29)$ siempre y $19,04 \%(\mathrm{n}=24)$ considera que siempre; así mismo, en cuanto a las presiones para salir como manifestación de conducta de acoso sexual, el 4,76\% ( $\mathrm{n}=6)$ opina que nunca, el 10,31\% $(\mathrm{n}=13)$ que casi nunca, el 30,15\% $(n=38)$ que a veces, el $26,98 \%(n=36)$ que casi siempre y el $27,77 \%(\mathrm{n}=35)$ que siempre; $\mathrm{y}$, finalmente relacionado con la conducta de invitar a salir con intenciones sexuales, el $3,17 \%(\mathrm{n}=4)$ opina que nunca debe ser considerada una conducta de acoso, el 6,34\% $(\mathrm{n}=8)$ que casi nunca, el 30,95\% $(\mathrm{n}=39)$ que a veces, el $28,57 \%(n=36)$ que muchas veces y el $30,95 \%(n=39)$ que siempre. 
1.4. Percepción de acoso sexual (Nivel 4, Acoso fuerte, con contacto físico)

\begin{tabular}{|l|c|c|c|c|c|}
\hline \multicolumn{7}{|c|}{ Tabla 4. Nivel I: Acoso grave, con contacto físico } \\
\hline & $\mathbf{0}$ & $\mathbf{1}$ & $\mathbf{2}$ & $\mathbf{3}$ & $\mathbf{4}$ \\
\hline $\begin{array}{l}\text { Besos, pellizcos, toca- } \\
\text { mientos }\end{array}$ & $\begin{array}{c}3,17 \% \\
(\mathrm{n}=4)\end{array}$ & $\begin{array}{c}5,55 \% \\
(\mathrm{n}=7)\end{array}$ & $\begin{array}{c}20,63 \% \\
(\mathrm{n}=26)\end{array}$ & $\begin{array}{c}32,53 \% \\
(\mathrm{n}=41)\end{array}$ & $\begin{array}{c}38,09 \% \\
(\mathrm{n}=48)\end{array}$ \\
\hline Sujetar o acorralar & $\begin{array}{c}5,55 \% \\
(\mathrm{n}=7)\end{array}$ & $\begin{array}{c}7,14 \% \\
(\mathrm{n}=9)\end{array}$ & $\begin{array}{c}15.07 \% \\
(\mathrm{n}=19)\end{array}$ & $\begin{array}{c}41,26 \% \\
(\mathrm{n}=52)\end{array}$ & $\begin{array}{c}30,95 \% \\
(\mathrm{n}=39)\end{array}$ \\
\hline $\begin{array}{l}\text { Acercamientos } \\
\text { vos }\end{array}$ & $\begin{array}{c}4,76 \% \\
(\mathrm{n}=6)\end{array}$ & $\begin{array}{c}7,93 \% \\
(\mathrm{n}=10)\end{array}$ & $\begin{array}{c}18,25 \% \\
(\mathrm{n}=23)\end{array}$ & $\begin{array}{c}34,92 \% \\
(\mathrm{n}=44)\end{array}$ & $\begin{array}{c}34,12 \% \\
(\mathrm{n}=43)\end{array}$ \\
\hline
\end{tabular}

0(nunca); 1(casi nunca); 2 (a veces); 3 (muchas veces); 4 (siempre)

Los resultados obtenidos en este nivel son: en relación a si la conducta de besar, pellizcar o tocar puede ser considerada una conducta acosadora, el 3,17\% $(\mathrm{n}=4)$ manifiesta que nunca, el 5,55\% $(\mathrm{n}=7)$ que casi nunca, el $20,63 \%(\mathrm{n}=269$ que a veces, el $32,53 \%(\mathrm{n}=41)$ que muchas veces y el $38,09 \%(\mathrm{n}=48)$ que siempre; con respecto a la conducta de sujetar o acorralar, el 5,55\% $(\mathrm{n}=7)$ manifiesta que nunca, el $7,14 \%(n=9)$ que casi nunca, el $15,04 \%(n=19)$ que a veces, el $41,26 \%(n=52)$ que muchas veces y el 30,95\% $(\mathrm{n}=39)$ que siempre; y finalmente, sobre los acercamientos excesivos, el 4,76\% $(n=6)$ opina que nunca, el 7,93\% $(n=10)$ que casi nunca, el $18,25 \%(\mathrm{n}=23)$ que a veces, el $34,92 \%(\mathrm{n}=44)$ que muchas veces y el $34,12 \%(n=43)$ que siempre.

\subsection{Percepción de acoso sexual (Nivel 5, Acoso grave)}

\begin{tabular}{|l|c|c|c|c|c|}
\hline \multicolumn{6}{|c|}{ Tabla 5. Nivel V: Acoso muy grave: contacto físico no deseado } \\
\hline & $\mathbf{0}$ & $\mathbf{1}$ & $\mathbf{2}$ & $\mathbf{3}$ & $\mathbf{4}$ \\
\hline Presiones físicas para & $4,76 \%$ & $7,14 \%$ & $11,90 \%$ & $37,30 \%$ & $38,88 \%$ \\
tener contactos íntimos & $(\mathrm{n}=6)$ & $(\mathrm{n}=9)$ & $(\mathrm{n}=15)$ & $(\mathrm{n}=47)$ & $(\mathrm{n}=49)$ \\
\hline Presiones psíquicas para & $7,14 \%$ & $8,73 \%$ & $26,98 \%$ & $30,15 \%$ & $26,98 \%$ \\
tener contactos íntimos & $(\mathrm{n}=9)$ & $(\mathrm{n}=11)$ & $(\mathrm{n}=34)$ & $(\mathrm{n}=38)$ & $(\mathrm{n}=34)$ \\
\hline Violación & $0 \%$ & $0 \%$ & $3,96 \%$ & $4,76 \%$ & $91,26 \%$ \\
& $(\mathrm{n}=0)$ & $(\mathrm{n}=0)$ & $(\mathrm{n}=5)$ & $\mathrm{n}=6$ & $\mathrm{n}=115$ \\
\hline
\end{tabular}

0 (nunca); 1(casi nunca); 2 (a veces); 3 (muchas veces); 4 (siempre)

Relacionada con las conductas más graves, en cuanto a las presiones físicas para tener contactos íntimos, el 4,76\% $(\mathrm{n}=6)$ opina que nunca, el 7,14\% $(\mathrm{n}=9)$ que casi nunca, el $11,90 \%(n=15)$ que a veces, el $37,30 \%(n=47)$ que muchas veces y el $38,88 \%(\mathrm{n}=49)$ que siempre; en relación a las presiones psíquicas para tener contactos físicos, el 7,14\% $(\mathrm{n}=9)$ manifiesta que nunca, el 8,73\% $(\mathrm{n}=11)$ que casi nunca, el $26,98 \%(\mathrm{n}=34)$ que a veces, el $30,15 \%(\mathrm{n}=38)$ que muchas veces y el $26,98 \%$ $(\mathrm{n}=34)$ que siempre; y finalmente, en cuanto a la violación, el 3,96\% $(\mathrm{n}=5)$ de los 
participantes considera que a veces podía considerarse una conducta de acoso, el $4,76 \%(n=6)$ que muchas veces y el $91,26 \%(n=115)$ que siempre.

\section{Percepción de acoso sexual en el trabajo en función del género}

A continuación se exponen los porcentajes diferenciadores entre hombres y mujeres en cada uno de los niveles de acoso. Los datos están realizados sobre el porcentaje total de cada uno, es decir, 77\% ( $\mathrm{n}=97)$ de mujeres y el 23\% (29) hombres:

\begin{tabular}{|c|c|c|c|c|c|c|}
\hline \multicolumn{7}{|c|}{ Tabla 6. Percepción acoso sexual en función del género } \\
\hline & & $\mathbf{0}$ & 1 & 2 & 3 & 4 \\
\hline \multirow[t]{3}{*}{$\begin{array}{l}\text { Acoso } \\
\text { leve }\end{array}$} & Hombres & $\begin{array}{c}27,58 \% \\
(\mathrm{n}=24)\end{array}$ & $\begin{array}{c}27,58 \% \\
(\mathrm{n}=24)\end{array}$ & $\begin{array}{c}18,39 \% \\
(\mathrm{n}=16)\end{array}$ & $\begin{array}{c}16,09 \% \\
(\mathrm{n}=14)\end{array}$ & $\begin{array}{c}10,34 \% \\
(\mathrm{n}=9)\end{array}$ \\
\hline & Mujeres & $\begin{array}{c}31,61 \% \\
(\mathrm{n}=92)\end{array}$ & $\begin{array}{c}30,24 \% \\
(\mathrm{n}=88)\end{array}$ & $\begin{array}{c}19,93 \% \\
(\mathrm{n}=58)\end{array}$ & $\begin{array}{l}11,68 \% \\
(\mathrm{n}=34)\end{array}$ & $\begin{array}{l}6,52 \% \\
(n=19)\end{array}$ \\
\hline & Total & $(n=116)$ & $(n=112)$ & $(\mathrm{n}=74)$ & $(n=48)$ & $(n=28)$ \\
\hline \multirow[t]{3}{*}{$\begin{array}{l}\text { Acoso } \\
\text { moderado }\end{array}$} & Hombres & $\begin{array}{l}18,96 \% \\
(\mathrm{n}=11)\end{array}$ & $\begin{array}{c}24,13 \% \\
(\mathrm{n}=14)\end{array}$ & $\begin{array}{c}27,58 \% \\
(\mathrm{n}=16)\end{array}$ & $\begin{array}{c}17,24 \% \\
(\mathrm{n}=10)\end{array}$ & $\begin{array}{c}12,06 \% \\
(n=7)\end{array}$ \\
\hline & Mujeres & $\begin{array}{l}2,57 \% \\
(\mathrm{n}=5)\end{array}$ & $\begin{array}{c}14,43 \% \\
(\mathrm{n}=28)\end{array}$ & $\begin{array}{c}31,95 \% \\
(\mathrm{n}=62)\end{array}$ & $\begin{array}{c}26,80 \% \\
(\mathrm{n}=52)\end{array}$ & $\begin{array}{c}24,22 \% \\
(n=47)\end{array}$ \\
\hline & Total & $(\mathrm{n}=16)$ & $(\mathrm{n}=42)$ & $(\mathrm{n}=78)$ & $(\mathrm{n}=62)$ & $(n=54)$ \\
\hline \multirow[t]{3}{*}{$\begin{array}{l}\text { Acoso } \\
\text { medio }\end{array}$} & Hombres & $\begin{array}{c}23,27 \% \\
(\mathrm{n}=27)\end{array}$ & $\begin{array}{c}35,34 \% \\
(\mathrm{n}=41)\end{array}$ & $\begin{array}{c}24,13 \% \\
(\mathrm{n}=28)\end{array}$ & $\begin{array}{l}9,48 \% \\
(\mathrm{n}=11)\end{array}$ & $\begin{array}{l}7,75 \% \\
(n=9)\end{array}$ \\
\hline & Mujeres & $\begin{array}{l}2,57 \% \\
(\mathrm{n}=10)\end{array}$ & $\begin{array}{l}4,12 \% \\
(\mathrm{n}=16)\end{array}$ & $\begin{array}{l}34,27 \% \\
(n=133)\end{array}$ & $\begin{array}{l}29,89 \% \\
(\mathrm{n}=116)\end{array}$ & $\begin{array}{l}29,12 \% \\
(n=113)\end{array}$ \\
\hline & Total & $(\mathrm{n}=37)$ & $(\mathrm{n}=57)$ & $(\mathrm{n}=161)$ & $(\mathrm{n}=127)$ & $(n=122)$ \\
\hline \multirow[t]{3}{*}{$\begin{array}{l}\text { Acoso } \\
\text { grave }\end{array}$} & Hor & $\begin{array}{l}13,79 \% \\
(\mathrm{n}=12)\end{array}$ & $\begin{array}{c}18,39 \% \\
(\mathrm{n}=16)\end{array}$ & $\begin{array}{c}17,24 \% \\
(\mathrm{n}=15)\end{array}$ & $\begin{array}{c}27,58 \% \\
(\mathrm{n}=24)\end{array}$ & $\begin{array}{c}22,98 \% \\
(\mathrm{n}=20)\end{array}$ \\
\hline & Mujeres & $\begin{array}{l}1,71 \% \\
(n=5)\end{array}$ & $\begin{array}{l}3,43 \% \\
(\mathrm{n}=10)\end{array}$ & $\begin{array}{l}18,21 \% \\
(\mathrm{n}=53)\end{array}$ & $\begin{array}{l}38,83 \% \\
(\mathrm{n}=113)\end{array}$ & $\begin{array}{l}37,80 \% \\
(\mathrm{n}=110)\end{array}$ \\
\hline & Total & $(\mathrm{n}=17)$ & $(\mathrm{n}=26)$ & $(\mathrm{n}=68)$ & $(\mathrm{n}=137)$ & $(n=130)$ \\
\hline \multirow{3}{*}{$\begin{array}{l}\text { Acoso } \\
\text { muy } \\
\text { grave }\end{array}$} & Hombres & $\begin{array}{l}12,64 \% \\
(\mathrm{n}=11)\end{array}$ & $\begin{array}{c}13,79 \% \\
(\mathrm{n}=12)\end{array}$ & $\begin{array}{c}22,98 \% \\
(\mathrm{n}=20)\end{array}$ & $\begin{array}{c}24,13 \% \\
(\mathrm{n}=21)\end{array}$ & $\begin{array}{c}26,43 \% \\
(\mathrm{n}=23)\end{array}$ \\
\hline & Mujeres & $\begin{array}{l}1,34 \% \\
(\mathrm{n}=4)\end{array}$ & $\begin{array}{l}2,74 \% \\
(\mathrm{n}=8)\end{array}$ & $\begin{array}{c}11,68 \% \\
(\mathrm{n}=34)\end{array}$ & $\begin{array}{c}24,05 \% \\
(\mathrm{n}=70)\end{array}$ & $\begin{array}{l}60,13 \% \\
(n=175)\end{array}$ \\
\hline & Total & $(\mathrm{n}=15)$ & $(\mathrm{n}=20)$ & $(\mathrm{n}=54)$ & $(\mathrm{n}=91)$ & $(n=198)$ \\
\hline
\end{tabular}

Como se puede observar en los datos obtenidos, y eludiendo ser reiterativos, cabe destacar las importantes diferencias en ambos géneros en referencia a todos los niveles de acoso. 


\section{Discusión}

En cuanto a los resultados obtenidos relacionados con el concepto de acoso sexual, parece claro que, en general, los participantes coinciden en una serie de características definitorias aportadas por diferentes autores y organizaciones referidas en el apartado introductorio de este trabajo tales como su carácter de naturaleza sexual, intimidatoria, indigna y ofensiva. Por tanto, quizás los programas de prevención que se deban plantear en este sentido, habrán de estar basados, sobre todo, en entender qué límites son los que determinarán cuales de estas conductas se encuentran dentro de las conductas delictivas de acoso, diferenciándolas de otras que se expliquen por la permisividad concedida en nuestra sociedad a determinadas conductas que se entienden como fruto de la galantería y caballerosidad de los hombres y a otras que se describen como conductas "atrevidas", groseras o de mal gusto, o malentendidas y/o malinterpretadas, pero sin llegar a considerarse constitutivas de acoso.

De la misma forma, además del atractivo físico, llama la atención como un porcentaje elevado de los participantes consideran que el acoso no es posible entre personas con igualdad de poder. Estos datos son de especial relevancia ya que, en general, se valora el poder que viene otorgado desde la jerarquía profesional obviando otro tipo de poder más relativo y, no por eso, menos relevante e influyente. Así mismo, en algunas ocasiones la autoridad ejercida por las personas con mayor estatus profesional se malinterpreta como conductas intimidatorias o de acoso moral. Por ello, insistimos en la necesidad de generar espacios formativos en donde se reflexione sobre las características de conductas hostiles.

Entre los datos obtenidos resaltamos la percepción que presentan algunos de los entrevistados con respecto a la responsabilización que se le otorga a la víctima sobre su capacidad de poder controlar la situación de acoso entendiendo que la persona podría liberarse del acosador empleando determinadas actuaciones. Estos resultados resaltan la falta de comprensión del fenómeno del acoso, obviando el proceso psicológico que padece la víctima, en donde quedan mermadas variables tan importantes como la autoestima, además del proceso emocional que sufre, no viéndose a si misma capaz de afrontar la situación para salir adecuadamente de la misma. Según Hirigoyen (2001), el 69\% de las personas entrevistadas víctimas del acoso laboral habían sufrido un trastorno depresivo mayor y el $52 \%$ presentaba trastornos psicosomáticos tales como problemas digestivos, cefaleas, trastornos del sueño, etc. Para esta misma autora, el acoso laboral deja unas huellas indelebles, pudiendo aparecer toda la sintomatología de un estrés postraumático así como un sentimiento de vergüenza continuo o incluso cambios en sus rasgos de personalidad. La víctima lleva una cicatriz psicológica que la hace frágil y la lleva a vivir durante un tiempo con cierta desconfianza hacia los demás. En nuestro caso de estudio, el acoso sexual, las consecuencias son iguales aunque habría que destacar que el sentimiento de vergüenza prevalece durante mucho tiempo debido a que se ha invadido el espacio más íntimo de la persona. Así mismo, el resto del entorno, influenciado por el agresor, criticará y pondrá en duda la parte de responsabilidad 
de la víctima alegando excusas como "tu le has dado pié", "eso era una broma", etc. Todo ello, favorecerá el desgaste emocional de la víctima y su predisposición a padecer diversas patologías psicológicas.

En relación a los resultados hallados en cuanto al perfil del agresor destacamos como un total de $63,48 \%$ considera que la persona acosadora es un hombre al que se le atribuyen características como ser vicioso, psicópata o adicto al sexo. De la misma forma, los participantes que no hacen distinción por sexo atribuyen dicha conducta a personas con mala intencionalidad en los sentimientos y las conductas, más concretamente "ser malas personas". Las investigaciones que aparecen en la literatura resaltan que no existe un único perfil de acosador. Así mismo, identifican como la mayoría de las personas acosadoras son hombres sin ninguna patología asociada pero que comparten una serie de características en común. Por un lado, no consideran a la mujer con la misma valía ni personal ni profesional recurriendo a las conductas sexuales como un medio para demostrar el poder y la superioridad profesional. Por otra parte, manifiestan patrones de comportamiento comunes, tales como alto grado de agresividad, falta de empatía, rasgos dominantes y conductas sexistas. De la misma forma, suelen ser personas con pareja, argumento que inicialmente utilizan para justificar su comportamiento de cortejo o coqueteo ante la víctima y que, igualmente, cuando es denunciado formal o informalmente, para justificar la falsedad de los hechos ante los demás. Y, finalmente, suelen ser personas con categoría profesional superior a la víctima, frecuentemente con cargos intermedios y que cuentan con la confianza de la empresa.

Siguiendo con el perfil de la víctima, un $73,79 \%$ considera que es mujer, destacando que un $34,12 \%$ lo asocia al atractivo de la misma. Estos datos son coincidentes con la creencia errónea de que existen personas con un perfil de víctimas y que éste guarda relación con los cánones de belleza de la sociedad. Otra creencia errónea es considerar que sólo las mujeres son víctimas de acoso sexual, ya que, como se ha apuntado en la introducción, si bien es cierto que la inmensa mayoría de personas acosadas son mujeres, esto no significa que no haya hombres que la sufren. Con respecto al atractivo físico, diversos estudios ponen en evidencia de la percepción que existe a nivel social sobre la correlación entre el atractivo y las conductas de acoso. Según los resultados obtenidos a partir de la investigación de Popovich et al. (1996), el atractivo físico, tanto del acosador como de la víctima, tendría efectos sobre la percepción de acoso sexual. Las personas tienden a responder basándose en ciertos procesos psicológicos como mitos, o sesgos cognitivos, como por ejemplo "el efecto halo" por el cual juzgamos a una persona a partir de un rasgo o cualidad. De esta forma, si una persona posee un aspecto positivo o negativo que destaca, trasladamos este aspecto a todos los rasgos. Así, por ejemplo, cuando una persona es atractiva tendemos a percibirla de una manera positiva en otros rasgos. Por ello, se tiende a favorecer a la víctima o al acosador más atractivo. Este estudio mostró que los hombres tienden a creer que el acoso se produce cuando la víctima es atractiva mientras que el acosador lo es poco. De la misma forma, si el hombre es atractivo, la relación con la víctima se tiende a percibir como más positiva. Los resultados hallados también revelan que las mujeres perciben la atracción 
sexual como motivo sólo cuando la víctima es atractiva, considerando irrelevante el atractivo del acosador. Estos hallazgos conllevan importantes consecuencias; por un lado, la creencia que un acosador atractivo tiene motivos más justificados para su comportamiento, mientras que cuando es poco atractivo es llevado por la atracción sexual; por otro lado, justifican cómo algunas conductas no son consideradas acoso, de este modo las mujeres que se perciben a sí misma como más atractivas entienden las conductas iniciales de acoso como meros acercamientos de coqueteo, mientras que las mujeres que se perciben así mismas como menos atractivas valoran desde el inicio esta conductas como acoso hacia ellas (Ellis, Barak y Pinto, 1991).

El atractivo físico es fuente de grandes influencias sociales, que en la mayoría de los casos favorecen a la persona considerada físicamente atractiva según los cánones de belleza de su cultura. Como indican (Popovich, P. y cols., 1996), estudiando los jurados en EEUU que intervienen en juicios relacionados con acoso sexual, se demostraba que cuando el juzgado es un hombre que posee características consideradas atractivas físicamente, y la víctima carece de ellas, el jurado suele ser más benévolo en su dictamen, que cuando la situación es a la inversa. Así mismo, en otros delitos, los jueces americanos eran menos propensos a encontrar a los imputados culpables cuando estos eran físicamente atractivos. Sin embargo, esto dependía con el tipo de delito por el que era juzgado. Así, los inculpados que eran considerados físicamente atractivos recibían un notable menor castigo que aquellos poco atractivos en los crímenes de robo, violación y engaño, pero recibían mayores castigos que aquellos físicamente poco atractivos en el homicidio negligente. Se podría explicar el hecho en relación a que las personas tienen expectativas más altas puestas sobre personas físicamente más atractivas, por lo tanto cuando fallan ante estas expectativas se le castiga más duramente (Mazzella, R. y Feingold, A., 1994).

Sin embargo, el problema del acoso tiene más que ver con las relaciones de poder que con las relaciones sexuales. En tal sentido no puede hablarse que existan víctimas típicas, sino más bien una asociación entre la probabilidad de ser víctima de acoso sexual y el grado de dependencia económica hacia el acosador, así como otros factores relacionados con el grado general de vulnerabilidad general de la persona. La Organización Internacional del Trabajo (OIT) (1997) señala que las mujeres con más probabilidad de ser acosadas son las viudas, separadas, divorciadas, mujeres que trabajan en trabajos predominantemente masculinos, mujeres recién ingresadas en la fuerza de trabajo y mujeres con contratos de empleo irregular.

Con respecto a los resultados reconocidos por los participantes, podemos observar como son 23 los casos percibidos y sólo 3 los casos comunicados, siendo además esta comunicación informal y dirigida a su red social más próxima. Estos datos corroboran otros estudios que indican que el número de denuncias de acoso sexual es minoritaría, existiendo una falta de visibilidad social del acoso sexual, debido sobre todo a la tendencia al ocultamiento y a circunscribirlo a la esfera del delito en los casos más graves, minimizando el acoso leve y restándole importancia. Son pocas las personas que se atreven a denunciarlo, en su mayor parte limitadas por el miedo a represalias en forma de despido o degradación laboral. Por otro lado, la 
culpabilización que mantienen por mucho tiempo las víctimas, pensando que podrían haber hecho algo y, la vergüenza que les produce haber estado sometidas a conductas vejatorias y no haber sido capaces de afrontarlas de otra manera, son otros motivos para el escaso grado de denuncias. En cuanto al resto de las personas del entorno laboral de la víctima, se convierten la mayoría de las veces en testigos mudos que, bien por su miedo a que pueda peligrar su puesto o a ver truncada su progresión profesional, bien porque comparten modos de actuación con el acosador, se posicionan a favor del mismo (Piñuel y Zabala, 2003). Así mismo, otras personas prefieren no ver para no tener que participar, y otras tranquilizan su conciencia ofreciendo soluciones a la víctima que le son imposibles de llevar a cabo. En cualquier caso, de una manera voluntaria o involuntaria, todos participan en el proceso de destrucción de la persona acosada. El apoyo social resulta esencial para el afrontamiento que la víctima realice sobre la situación que esta sufriendo, sin embargo, los datos revelan lo que en su mayor parte y hasta la fecha es lo habitual. La víctima se siente el centro de "los rumores de los pasillos y de los encuentros del café". Se comenta, se critica y se pone en tela de juicio en muchos casos incluso características físicas y conductas de la víctima del tipo "mira como se maquilla", "se pone una ropa muy apretada", "se ríe con los chistes de sexo", "yo creo que ha tenido un lío y le ha salido mal", etc. Además del proceso por el que está pasando, a menudo la víctima pasa a sufrir un juicio popular en el cual se siente poco defendida y apoyada y que para nada facilita su afrontamiento de la situación.

La menor importancia concedida por parte de los hombres a las conductas verbales como piropos, chistes o comentarios como constitutivas de acoso, podría explicarse, según los estudios de Ardouin, Bustos, Jarpa y Oliveros (2001), por rasgos culturales en los que el hombre malinterpreta algunas conductas atribuyéndole intenciones sexuales por parte de la mujer. Nuestra cultura está inmersa en patrones machistas, siendo habitual en reuniones de hombres el piropo, los chistes o comentarios hacia mujeres. De la misma forma, estos mismos comportamientos, aunque cada vez en menor medida, aún siguen estando censurados en las reuniones de mujeres, considerándolos no deseables socialmente, atribuyéndoles ellas mismas a congéneres que realizan estos comentarios hacia hombres adjetivos como "ligeras de cascos", "provocadoras", "ordinarias", etc. Es importante reflexionar sobre esta cuestión, ya que cuando una de estas mujeres sufre acoso, se producen dos reacciones habituales del entorno. Por un lado, de incredulidad, ya que se les consideran mujeres con adecuadas estrategias de afrontamiento a este tipo de situaciones, o bien, de castigo, manifestando que "se lo merece", ya que su conducta genera situaciones que llevan a la provocación y por tanto, "ahora que no se queje", ya que "ella misma lo ha querido as'́".

De la misma forma, los datos obtenidos en cuanto a los piropos $(33,33 \%(\mathrm{n}=42$; nunca y $34,9 \%(n=44)$, casi nunca $)$ pueden explicarse, al igual que los chistes y comentarios, debido al contexto cultural en el que el piropo formaba parte de la socialización del hombre y del modelo de masculinidad. Era utilizado como una manifestación habitual del cortejo en donde se expresaba el sentimiento de atracción hacia una mujer. Siguiendo a Correa y Mancera (2009), esta práctica ya no es 
sólo un medio por el que se conquista y se halaga a una mujer, sino que actualmente se considera una práctica por la que el hombre puede llegar a hacer que la mujer se sienta insultada y denigrada. Desde la reflexión de este trabajo, y entendiendo la relevancia que este tipo de conductas tiene en los procesos de acoso, lo importante sería encontrar la línea divisoria entre el halago y el piropo, y, evidentemente no sólo en el qué se dice sino en cómo se dice, esa comunicación no verbal tan sutil empleada por muchos de los acosadores que minan día a día la dignidad de la persona que han elegido como víctima.

En relación a los resultados obtenidos referentes al Nivel II de acoso moderado, aquel que tiene sus conductas manifiestas en miradas y gestos insinuantes, se puede observar como la mayoría de los sujetos de la muestra indican que carecen de importancia y, difícilmente podrían denominarse acoso. Estos datos vuelven a manifestar el amplio espectro de situaciones y conductas constitutivas de acoso sexual, así como la falta de información por parte de la sociedad en relación a este grave problema. Es evidente, que conductas que impliquen menor cercanía física del acosador hacia la víctima, se valora como menos violadora de nuestro espacio personal. Sin embargo, es necesario concienciar que este repertorio verbal y no verbal caracterizado por insinuaciones, dobles sentidos, miradas desafiantes, etc, puede ser el inicio de un ambiente hostil y debe ser detectado y paliado desde el principio ya que en la mayoría de los casos es uno de los primeros indicios de acoso.

Continuando con el Nivel III se observa la menor importancia que obtienen algunas conductas como los envíos de correos o las cartas, aumentando su importancia en las conductas que implican más presión e insinuaciones, dando como resultado una mayor intimidación de la víctima. Estos resultados, al igual que explicábamos anteriormente, podrían reflejar, por un lado, la sensación de una mayor capacidad de control y seguridad ya que las cartas y correos no implican la cercanía con el agresor, y por otro lado, a medida que las conductas son más intimidatorias la persona las percibe como más amenazantes.

Por la gravedad de los hechos, debemos detenernos a analizar el número aunque pequeño de personas $(n=5)$ que consideran que la violación pudiera a veces estar justificada. Como comentábamos en la descripción del instrumento, en el apartado comentarios, las personas podían expresar cuanto quisieran o aclarar las respuestas que habían aportado. Todas las personas eran hombres y dieron esta respuesta justificando dicha actuación en el comportamiento provocador de la víctima que lo podía llevar a una falta de control del instinto sexual primario. Resulta sorprendente que todavía se continúe, por parte de un nada despreciable número de personas, planteando una explicación biologicista a conductas tan graves de agresión sexual, basadas en la imposibilidad de controlar impulsos sexuales en respuesta a estímulos basados en la atracción sexual.

En cuanto a la diferenciación por género, se puede observar como los porcentajes se encuentran más polarizados cuando hablamos de conductas sexuales leves, no siendo así cuando se avanza en la gravedad del mismo en donde parece existir un consenso mayor en cuanto a lo que se pueden considerar como conductas acosadoras. 
Con relación a la diferencia de percepción de situaciones de acoso entre las personas empleadas y desempleadas, no hemos expuesto los datos ya que los resultados pudieran resultar poco significativos, por cuanto que un número relativamente importante de las personas que trabajaban también se encontraban formándose y viceversa. En cualquier caso, los datos generales indican una mayor concienciación por parte de las personas que se encuentran empleadas con respecto a la mayor parte de las conductas que se consideran en la bibliografía como situaciones de acoso. La explicación la podemos encontrar en que quizás las personas que se encuentran inmersas en el mundo laboral han sido testigos o víctimas de conductas parecidas a las presentadas en la evaluación. La inmersión en el mundo laboral les ha permitido ser más conscientes de la existencia de estas y otras situaciones que se presentan con demasiada frecuencia en el ámbito de las relaciones laborales y por tanto, son más conocedoras de la gravedad de los actos que se les exponían en el cuestionario.

\section{Referencias bibliográficas}

Ardouin J.; Bustos C.; Jarpa M. y Oliveros G. (2001). Acoso sexual: una monografía de psicología social aplicada al acoso sexual. Recuperado el 3 de Enero, 2011, de http://www.apsique.com/wiki/SociAcoso

Barak, A., Fisher, W.A. y Huston, S. (1992). Individual difference correlates of the experience of sexual harassment among female university students. Journal of Applied Social Psycholog. Vol. 22, 17-37.

Castillo, A. (1997) El acoso sexual en el trabajo y en los centros de estudio: flagelo a derrotar. Revista Venezolana de Estudios de la Mujer. Vol. 2, Núm. 5.

Correa y Mancera (2009). La práctica del piropo en los trabajadores de la construcción que laboran en la ciudad de Bogotá. Bogotá: Pontificia Universidad Javeriana.

Comisión de Igualdad de Oportunidades de Empleo de Estados Unidos (EEOC) (1980). Guias sobre el Hostigamiento Sexual y el Empleo. US: EEOC

Ellis, S., Barak, A. y Pinto A. (1991). Moderating effects of personal cognitions on experienced and perceived sexual harassment of woman at the workplace. Journal of Applied Social Psychology. Vol. 21, 1320-1337.

Fitzgerald, L.F., Drasgow, F., Hulin, C.L., Gelfand, M.J. y Magley, V.J. (1997). Antecedents and consequences of sexual harassment in organizations: A test of an integrated model. Journal of Applied Psychology, Vol. 82 Núm. 4, 578-590. 
Hirigoyen (2001). El acoso moral en el trabajo: distinguir lo verdadero de lo falso. Barcelona: Paidós Ibérica.

Livingston, J. (1982). Responses to sexual harassment on the job: legal, organizational and individual actions. Joumal of Social Issues. Vol. 38, 5-22.

Menon, S. \& Kanekar, S. (1992). Attitudes toward sexual harassment of women in India. Journal of Applied Social Psychology. Vol. 22, 1940-1958

Mazzella, R., y Feingold, A. (1994). The effects of physical attractiveness, race, socioeconomic status, and gender of defendants and victims on judgments of mock jurors. Journal of Applied Social Psychology. Vol. 24, 1315-1344.

OIT (1997). Desagradable, no deseado y cada vez más ilegal: el acoso sexual en el lugar de trabajo. Revista Trabajo. Vol. 19, 24-29.

Pernas, B., Olza, J. y Román, M. (2000). El acoso sexual en el trabajo en España. Madrid: Secretaría Confederal de la Mujer de CC.OO. Recuperado el 3 de Enero, 2011, de http://www.ccoo.es/comunes/temp/recursos/1/643319.pdf

Piñuel y Zabala, I. (2003). Mobbing. Cómo sobrevivir al acoso psicológico en el trabajo. Santander: Sal Terrae.

Popovich, P., Gehaulf, D., Jelton, J., Everton, W., Godinho, R., Mastrangelo, P. \& Somers, J. (1996). Physical attractiveness and sexual harassment: does every picture tell a story or every story draw a picture?. Journal of Applied Social Psychology. Vol. 26, 520-542.

Stockdale, J.E. (1996). Sexual Harassment in the Workplace; Perspectives,Frontiers and Response Strategies. California: Sage Publications.

Tangri, S.S., Burt, M.R., y Johson, L.B. (1982). Sexual harassment at work: Three explanatory models. Journal of Social Issue. Vol. 38, 33-54.

Unión General de Trabajadores (1994). Guía sindical sobre acoso sexual en el trabajo. Madrid: UGT-Departamento de la Mujer.

Unión del Personal Civil de la NACIÓN (UPCN) (1997). Violencia laboral. Estudio sobre acoso Sexual. Buenos Aires: Secretaría de la mujer de UPCN. 